\title{
Fibrillin expression and localization in various types of carcinomas of the thyroid gland
}

\author{
Sofia Tseleni-Balafouta ${ }^{1}$, Hariklia Gakiopoulou ${ }^{1}$, Galinos Fanourakis ${ }^{1}$, \\ Gerassimos Voutsinas ${ }^{2}$, Helen Litsiou ${ }^{1}$, Elias Sozopoulos ${ }^{1}$, \\ Dimitrios Balafoutas ${ }^{1}$ and Efstratios Patsouris ${ }^{1}$ \\ ${ }^{1}$ Department of Pathology, Medical School University of Athens, Athens, Greece and ${ }^{2}$ Laboratory of \\ Environmental Mutagenesis and Carcinogenesis, Institute of Biology, NCSR 'Demokritos', Athens, Greece
}

\begin{abstract}
Fibrillin is an extracellular matrix (ECM) glycoprotein, a main component of microfibrills, suggested to support cell attachment and to impact cell differentiation and migration. The aim of this study was to investigate fibrillin1 expression in thyroid carcinomas at mRNA and protein level, since ECM proteins are suggested to be of great importance for the metastatic potential of carcinomas. RNA was extracted from 13 thyroid carcinoma cell lines and RT-PCR analysis with gene-specific primers revealed fibrillin-1 mRNA expression in all cell lines, with highest expression in the follicular carcinoma cell line WRO and lowest expression in the two anaplastic cell lines (APO, FRO). Furthermore, we investigated fibrillin-1 expression by immumohistochemistry in a commercially available tissue microarray including $\mathbf{5 0}$ thyroid carcinomas as well as in archival tissue from 33 thyroid carcinomas. Fibrillin-1 demonstrated a cytoplasmic location in the neoplastic cells of almost all carcinomas apart from the follicular ones. The most intense staining was observed in papillary carcinomas with some evidence of a slight increased intensity in advanced stages. Our data indicate that fibrillin-1 is strongly expressed by the neoplastic cells of thyroid carcinomas in different degree in the various histologic types and might be implicated in cell-stroma interaction in terms of signaling, attachment and migration.

Modern Pathology (2006) 19, 695-700. doi:10.1038/modpathol.3800578; published online 10 March 2006
\end{abstract}

Keywords: fibrillin-1; RT-PCR; thyroid carcinomas; tissue microarray; extracellular matrix

Establishment of an invasive and metastatic tumor phenotype depends on diverse and intricately orchestrated cell-cell and cell-matrix adhesive interactions. $^{1-3}$ In the case of epithelial cancers, the development of invasion and metastasis is currently regarded as a multistep process with interactions between migrating tumor cells, diverse extracellular matrices and activated endothelia. ${ }^{1-3}$

Fibrillin is a $350-\mathrm{kDa}$ glycoprotein which constitutes the main component of microfibrils-filamentous structures of small diameter (10-12 nm), found in the extracellular matrix (ECM) of most tissues. ${ }^{4-6}$ Although usually associated with elastin to form elastic fibers, microfibrils devoid of elastin are also found in the ECM of nonelastic tissues. ${ }^{7}$ It is likely that fibrillin has many functions that vary according to the developmental stage and tissue in which it is expressed., ${ }^{7,8}$ Of the known microfibrillar proteins,

Correspondence: Dr S Tseleni-Balafouta, MD, PhD, Department of Pathology, Medical School University of Athens, M. Asias 75, Athens 11527, Greece.

E-mail: stseleni@cc.uoa.gr

Received 10 January 2006; revised 1 February 2006; accepted 5 February 2006; published online 10 March 2006 fibrillins are the best candidates for interacting with cell surface receptors. ${ }^{9}$ Fibrillins contain Arg-GlyAsp (RGD) peptide sequence shown to be the site of interaction with integrin receptors. ${ }^{10,11}$ Along with integrin-ligand interactions, another major mode of cell-matrix interaction employs cell-surface heparan-sulphated proteoglycans (HSPGs) such as members of the syndecan or glypican families. ${ }^{12}$ Recently, Ritty et al ${ }^{13}$ suggested that cell attachment to fibrillins can potentially produce simultaneous signaling from members of these two ECM receptor families: the integrins and the cell-surface HSPGs. Thus, fibrillin may act as a classical adhesion protein with specific interactions with the cellular cytoskeleton involving transmembrane receptors. ${ }^{9}$ Moreover, given the functional roles of integrins and HSPGs it is already clear that fibrillins play important roles in cell migration, adhesion, signaling, protein-protein interaction and induction of differentiation. ${ }^{13}$ As all these procedures are also important during neoplastic transformation and tumor progression, the investigation of fibrillin expression in tumorous conditions seems challenging. 
Fibrillin has been shown to have a widespread distribution in the ECM of skin, lung, kidney, liver, blood vessels, tendons and ocular tissues. $^{4}$ Very little information is available as far as fibrillin expression in a neoplastic context is concerned. To the best of our knowledge, this is the first study so far, investigating fibrillin expression at protein and m-RNA level in thyroid carcinoma tissues and cell lines as well as in normal thyroid tissues.

\section{Materials and methods}

\section{Studies on Cell Lines and Cultures}

Thyroid cancer cell lines TT (medullary carcinoma), ARO, FRO (anaplastic carcinoma), WRO (follicular carcinoma), BHP 2, BHP 5, BHP 7, BHP 10, BHP 14, BHP 17, BHP 18, BHP 19 and SW579 (papillary carcinoma) were cultured in Dulbecco's minimal essential medium supplemented with $10 \%$ fetal bovine serum and $100 \mu \mathrm{g} / \mathrm{ml}$ penicillin/streptomycin (Life Technologies Inc.). At 80\% confluence, the medium was replaced with serum-free DMEM; the cells were incubated for $24 \mathrm{~h}$ and then harvested for mRNA extraction.

Isolation of total RNA and reverse transcription-PCR Total RNA from thyroid cancer cell lines was isolated using the Rneasy Kit (Qiagen) following the manufacturer's suggestions. Total RNA (1 $\mu \mathrm{g})$ was reverse transcribed to complementary DNA (cDNA) with SuperScript II reverse trascriptase (Life Technologies Inc.) primed with oligo-dT in a final concentration of $20 \mathrm{pmol} / \mu \mathrm{l}$. A ribonuclease inhibitor (Ribonuclease Inhibitor, Fermentas), in a final concentration of $1 \mathrm{U} / \mu \mathrm{l}$ was also included in the reaction. All RT reactions were done independently of the PCR reactions. Amplification of fibrillin-1 transcript was performed using genespecific primers (forward 5'-CTTGAAGGGAGAA GGCTGG-3', reverse 5'-AGGGACACTCGCAGCGA TA-3') producing a $205 \mathrm{bp}$ product. Briefly, $1 \mu \mathrm{l}$ cDNA was amplified with $10 \mathrm{pmol} / \mu \mathrm{l}$ primers using the puRe Taq Ready-To-Go PCR Beads (Amersham Biosciences) in a final volume of $25 \mu \mathrm{l}$. After initial denaturation at $95^{\circ} \mathrm{C}$ for $3 \mathrm{~min}$, amplification was performed over 30 cycles consisting of $95^{\circ} \mathrm{C}$ for $30 \mathrm{~s}, 55^{\circ} \mathrm{C}$ for $30 \mathrm{~s}, 72^{\circ} \mathrm{C}$ for $30 \mathrm{~s}$, and a final extension at $72^{\circ} \mathrm{C}$ for $7 \mathrm{~min}$. Negative controls were performed with each RT-PCR reaction, omitting template. The efficiency of reverse transcription was verified by the detection of the GAPDH (glyceraldehyde phosphate dehydrogenaseforward: $5^{\prime}$-TGGTATCGTGGAAGGACTCATGAC-3', reverse: $\quad 5^{\prime}$-ATGCCAGTGAGCTTCCCGTTCAG-3'), as previously described. ${ }^{14}$ The products were resolved on a $2 \%$ agarose gel containing ethidium bromide and were visualized under ultraviolet light.

\section{Densitometry}

The band intensity on PCR photographs was quantitated by densitometry using the GDS 8000 Analysis System (UVP). The intensity of the band representing the Fbn-1 RT-PCR product was normalized to the intensity of the band for the housekeeping gene GAPDH and is expressed as ratio of relative band intensity. All experiments were repeated at least three times to ensure statistical accuracy.

\section{Studies on Thyroid Specimens}

A total of 92 thyroid specimens from normal and neoplastic glands were investigated immunohistochemically for the detection of fibrillin-1 expression. This series consisted of 59 thyroid tissues from a commercially available tissue microarray (Imgenex IMH 319) including 42 papillary carcinomas, seven follicular carcinomas, one poorly differentiated carcinoma and nine benign thyroid tissues. Moreover, archival tissue from 33 thyroid carcinomas (15 papillary, four follicular, five Hüerthle cell-, one anaplastic and eight medullary carcinomas) was retrieved from the files of the Pathology Department of the Athens University. The clinicopathologic characteristics of our cases are shown in Table 1. Representative tissue blocks were selected from each thyroid specimen, for immunohistochemical examination (two blocks per case). Sections $(4 \mu \mathrm{m})$ were cut from paraffinembedded blocks.

\section{Fibrillin-1 immunohistochemistry}

Immunostaining was performed with a polyclonal antibody (Santa Cruz H-109) at a dilution of 1:100, overnight. For better antigen retrieval, tissue sections were microwave treated in citrate buffer, $\mathrm{pH}$ 6.0 , for $30 \mathrm{~min}$. A standard two-step streptavidin peroxidase technique was used. Sections from liver cirrhosis with known immunopositivity for fibrillin-1 antibody were used as positive controls. Negative controls had the primary antibody omitted and replaced by PBS.

Table 1 Clinicopathologic characteristics of patients with thyroid carcinomas

\begin{tabular}{lc}
\hline$N$ & 83 \\
Age (range) & $15-72$ \\
Male/female & $26 / 57$ \\
& \\
Histologic type & $(T M / A S)$ \\
Papillary & $57(42 / 15)$ \\
Follicular & $11(7 / 4)$ \\
Huerthle cell & $5(0 / 5)$ \\
Medullary & $8(0 / 8)$ \\
Poorly differentiated & $1(1 / 0)$ \\
Anaplastic & $1(0 / 1)$ \\
\hline
\end{tabular}

TM: tissue microarrays; AS: archival surgical specimens. 


\section{Evaluation of immunohistochemistry}

The sections were evaluated without knowledge of clinical data by two independent observers and similar results were obtained. A semiquantitative evaluation of fibrillin-1 expression was performed by means of scoring staining intensity, evaluating simultaneously both intensity and extension, on a scale of 0 to +++ , corresponding to negative (0), weak $(+)$, moderate $(++)$ and strong $(+++)$ immunoreactivity. The findings were then correlated to the tumor stage.

\section{Results}

\section{RT-PCR Analysis of Fibrillin-1 in Thyroid Carcinoma Cell Lines}

RT-PCR analysis revealed fibrillin-1 m-RNA expression in all thyroid carcinoma cell lines, with highest expression in the follicular carcinoma cell line WRO, the papillary carcinoma cell line SW579, and the medullary TT, whereas lowest expression was found in the two anaplastic cell lines (APO, FRO) and one papillary line (BHP 18) (Table 2, Figure 1).

\section{Fibrillin-1 Immunohistochemical Expression}

A weak fibrillin-1 immunoreactivity was observed in normal thyroid tissues mainly in the vessel wall as well as in the perivascular ECM as thin bundles. No fibrillin expression has been demonstrated

Table 2 RT-PCR analysis of fibrillin-1 mRNA expression in thyroid carcinoma cell lines

\begin{tabular}{llc}
\hline Cell line & Type & Fibrillin-1: GAPDH \\
\hline TT & Medullary & 0.965 \\
ARO & Anaplastic & 0.749 \\
FRO & Anaplastic & 0.860 \\
WRO & Follicular & 1.003 \\
BHP 2 & Papillary & 0.999 \\
BHP 5 & Papillary & 0.963 \\
BHP 7 & Papillary & 0.967 \\
BHP 10 & Papillary & 0.960 \\
BHP 14 & Papillary & 0.969 \\
BHP 17 & Papillary & 0.949 \\
BHP 18 & Papillary & 0.526 \\
BHP 19 & Papillary & 0.932 \\
SW 579 & Papillary & 0.999 \\
\hline
\end{tabular}

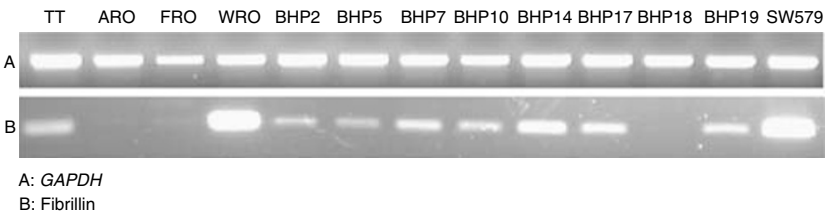

Figure 1 RT-PCR analysis of fibrillin expression in 13 thyroid Ca cell lines. in the perifollicular area. In thyroid carcinomas, fibrillin-1 immunohistochemical expression has been observed in the neoplastic cells with an intracytoplasmic location as well as in bundles in the stroma underneath (Figures 2 and 3). In the tissue microarray as well as in the archival sections fibrillin-1 expression was seen in almost all papillary carcinomas (39/42 of archival carcinomas and $14 / 15$ of tissue micrarray carcinomas respectively), Hüerthle cell carcinomas (4/5), and in all anaplastic (1/1) and poorly differentiated (1/1) carcinomas as well as in many medullary carcinomas $(5 / 8)$ (Table 3). The most intense staining was observed in papillary carcinomas, with a slightly increased intensity in advanced stages (Table 4). In the follicular carcinomas no fibrillin expression could

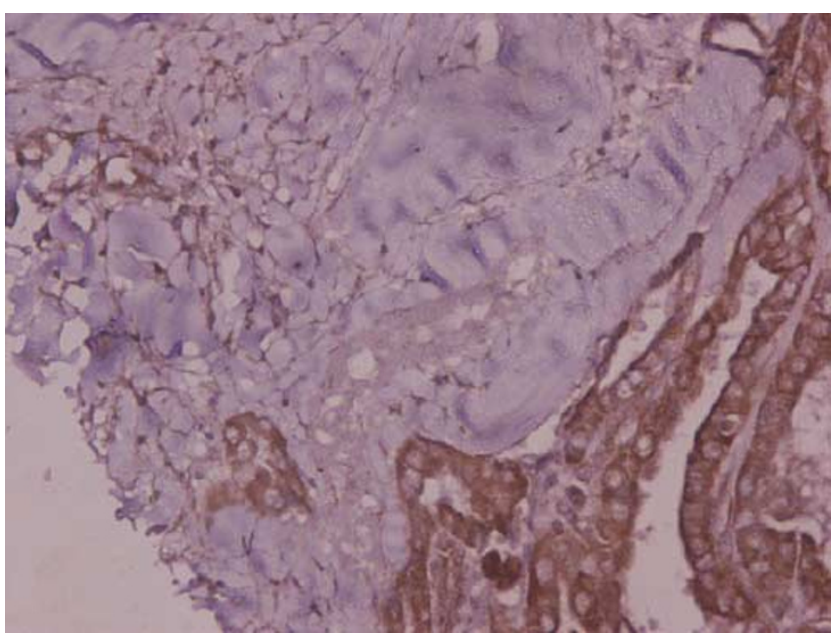

Figure 2 Fibrillin-1 immunohistochemical expression in a papillary thyroid carcinoma: strong immunoreactivity in the cytoplasm of neoplastic cells and weak immunoreactivity in thin bundles in the stroma underneath $(\times 200)$.

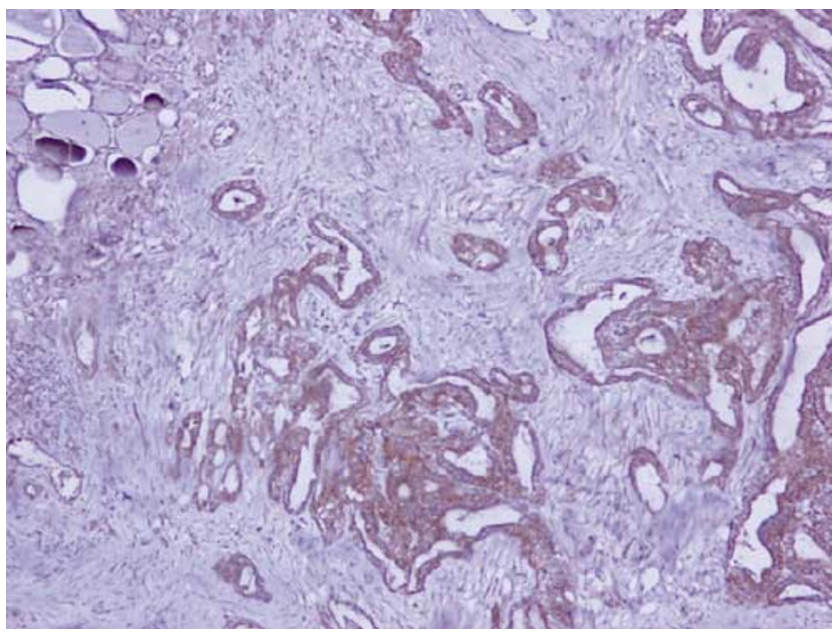

Figure 3 Fibrillin-1 immunohistochemical expression in a papillary thyroid carcinoma. Note the absence of cytoplasmic expression in normal follicles $(\times 100)$. 
Table 3 Immunohistochemical demonstration of fibrillin-1 expression in thyroid carcinoma tissue microarrays (a) and surgical specimens (b)

\begin{tabular}{|c|c|c|c|c|c|}
\hline \multirow[t]{2}{*}{ Histologic type } & \multirow[t]{2}{*}{$\mathrm{n}$} & \multicolumn{4}{|c|}{ Staining intensity } \\
\hline & & - & + & ++ & +++ \\
\hline \multicolumn{6}{|l|}{ (a) Tissue microarrays } \\
\hline Papillary Ca & 42 & $1(23.8 \%)$ & $17(40.4 \%)$ & $13(30.9 \%)$ & $11(26.2 \%)$ \\
\hline Follicular Ca & 7 & $7(100 \%)$ & $0(0 \%)$ & $0(0 \%)$ & $0(0 \%)$ \\
\hline Poorly differentiated Ca & 1 & $0(0 \%)$ & $0(0 \%)$ & $1(100 \%)$ & $0(0 \%)$ \\
\hline Benign thyroid tissue & 9 & $9(100 \%)$ & $0(0 \%)$ & $0(0 \%)$ & $0(0 \%)$ \\
\hline \multicolumn{6}{|l|}{ (b) Surgical specimens } \\
\hline Papillary Ca & 15 & $1(7 \%)$ & $0(0 \%)$ & $4(26 \%)$ & $10(67 \%)$ \\
\hline Follicular Ca & 4 & $4(100 \%)$ & $0(0 \%)$ & $0(0 \%)$ & $0(0 \%)$ \\
\hline Huerthle-cell Ca & 5 & $1(20 \%)$ & $1(20 \%)$ & $3(60 \%)$ & $0(0 \%)$ \\
\hline Anaplastic $\mathrm{Ca}$ & 1 & $0(0 \%)$ & $0(0 \%)$ & $1(100 \%)$ & $0(0 \%)$ \\
\hline Medullary Ca & 8 & $3(37.5 \%)$ & $4(50 \%)$ & $1(12.5 \%)$ & $0(0 \%)$ \\
\hline
\end{tabular}

Table 4 Correlation of fibrillin-1 expression with stage in thyroid papillary carcinomas (tissue microarrays and archival specimens)

\begin{tabular}{llcrr}
\hline \multirow{2}{*}{ Stage } & \multicolumn{4}{c}{ Fibrillin-1 expression } \\
\cline { 2 - 5 } & \multicolumn{1}{c}{0} & + & ++ & +++ \\
\hline I $(n=28)$ & $0(0 \%)$ & $11(39 \%)$ & $9(32 \%)$ & $8(29 \%)$ \\
II $(n=4)$ & $1(25 \%)$ & $2(50 \%)$ & $0(0 \%)$ & $1(25 \%)$ \\
III $(n=23)$ & $0(0 \%)$ & $4(17 \%)$ & $7(31 \%)$ & $11(52 \%)$ \\
IV $(n=2)$ & $1(50 \%)$ & $0(0 \%)$ & $1(50 \%)$ & $0(0 \%)$ \\
\hline
\end{tabular}

be detected in the immunohistochemically processed archival as well as tissue microarray sections (total 11 cases).

\section{Discussion}

In the present study, we describe for the first time, the distribution of the ECM protein fibrillin-1, in normal thyroid tissue and in thyroid carcinomas of various histological types. In our study, fibrillin-1 immunoreactivity was very faint in normal thyroid tissue and exclusively located in the perivascular area. No fibrillin-positive fibers have been observed between the thyroid follicles. Notably, this ECM protein was detected by immunohistochemistry in the cytoplasm of neoplastic cells of thyroid carcinomas and to a lesser extend in the surrounding stroma. This finding confirms previous experimental studies reporting that apart from fibroblastic cells, epithelial cells are responsible for fibrillin-1 production. ${ }^{13}$ ECM deposition occurs in cultures of many cell types including normal thyroid cells. ${ }^{15,16}$ The weak immunoreactivity of fibrillin-1 in the stroma of thyroid tumors suggests a mechanism probably inhibiting the deposition of the protein in the tumorous stroma, since aberrant deposition of matrix proteins has been reported in many tumor types. This might be especially important for papillary carcinoma which exhibits a high degree of elastosis, ${ }^{17}$ inasmuch as fibrillin-1 has been suggested to be colocalized with elastin in various tissues. ${ }^{8,18}$ Although the mechanism of fibrillin-1 deposition in ECM is largely unknown, it has been shown that in epithelial cells, soluble heparin competitively inhibits an unknown interaction that is needed for ECM deposition of both fibrillin-1 and $-2 .{ }^{13}$ Moreover, a human fibrillin-1 mutation (Arg ${ }^{2726} \rightarrow$ Trp) which prevents proteolytic processing of the C-terminal domain, has been reported to result in impaired deposition of the product of the affected allele. ${ }^{19}$

From a functional perspective, the possible role of fibrillin-1 localization in the neoplastic thyroid cells merits further investigation. The distribution of fibrillin-1 and fibrillin-2 in developing tissues suggests that both proteins are usually, but not exclusively, associated with elastic fibers. ${ }^{20}$ Colocalization of fibrillin-1 with $\mathrm{FC}_{\mathrm{C}}$ gamma RIII, a cytokine-inducible IgG Fc receptor implicated in the activation of macrophages by immune complexes, suggests a possible role of fibrillin-1 in the modulation of immunologic responces. ${ }^{21}$ It has been reported that at sites of inflammation or active tissue remodeling such as the neoplastic stroma, elastases could strip elastin from microfibrils, thereby exposing fibrillin molecules that are otherwise masked. ${ }^{9}$ An invading inflammatory cell with the appropriate receptors could then use fibrillin to move through an elastin-rich tissue such as a blood vessel. ${ }^{9}$ Proportionally, one could speculate that an invasive tumor cell expressing fibrillin-1 is able to use this protein as a vehicle to migrate through the tumorous stroma and/or invade vessels to produce metastases. This concept is strengthened by that observed in our study, slightly stronger fibrillin-1 expression in higher stage tumors (Table 4). This relation emanated mainly from the comparison of stages I and III where most 
of our cases were distributed (Table 4). From this point of view, a plausible association could also be expected between strong fibrillin-1 expression and anaplastic carcinoma given its well-established invasive potential. However, this concept was not confirmed in the unique case of anaplastic carcinoma investigated in our study suggesting that a fibrillin-1-independent invading mechanism might also exist or that a highly undifferentiated carcinoma has possibly lost the ability of fibrillin-1 expression.

An interesting and partly controvercial finding of our study lies on the comparison between our immunohistochemical and RT-PCR analysis, results.

Both methods detected fibrillin-1 protein and m-RNA, respectively, in the cytoplasm of neoplastic cells of most thyroid carcinomas investigated. A discrepancy was observed concerning follicular carcinomas in which RT-PCR demonstrated the highest expression of fibrillin-1 m-RNA while immunohistochemistry failed to detect the protein in archival surgical specimens as well as in tissue microarray sections of this tumor type. This finding implies that neoplastic follicular cells, while equipped with the genetic machinery to synthesize fibrillin-1, are unable to finally produce the protein probably due to post-transcriptional modifications. Alternatively, fibrillin-1 protein is expressed in such a low level, which might be undetectable by immunohistochemistry. Of note also that, given a putative common origin of papillary and follicular neoplasms from follicular cells, one could expect a similar behavior of these two histological types at a molecular level. However, we have already observed a similar discrepancy, concerning an other ECM protein, tenascin- $1 .{ }^{22}$ Our results are in line with the concept supported by several authors, that papillary and follicular neoplasms consist two genetically distinct types of thyroid neoplasms. ${ }^{23}$

In conclusion, we have described for the first time in normal thyroids and thyroid carcinomas, the expression of fibrillin-1 mRNA and the localization of fibrillin-1 protein. Our RT-PCR and immunohistochemical results indicate that fibrillin-1 is produced by thyroid carcinoma cells with evidence of an increased protein intensity in the more advanced stage carcinomas. A possible role of fibrillin-1 in thyroid cancer progression probably by facilitating access of tumor cells in the elastin-rich blood vessel walls or through the elastin-rich stroma of thyroid carcinomas could be suggested.

\section{Acknowledgements}

We thank Dr N Mitsiades, Dana Farber Cancer Institute Harvard Medical School, for providing the thyroid carcinoma cell lines used in this study.

\section{References}

1 Koukoulis GK, Patriarca C, Gould VE. Adhesion molecules and tumor metastasis. Hum Pathol 1998; 29:889-892.

2 Murphy-Ullirich JE. The de-adhesive activity of matricellular proteins: is intermediate cell adhesion an adaptive state? J Clin Invest 2001;7:785-790.

3 Juliano RL, Haskill S. Signal transduction from the extracellular matrix. J Cell Biol 1993;120:577-585.

4 Dubuisson L, Lepreux S, Bioulac-Sage P, et al. Expression and cellular localization of fibrillin-1 in normal and pathological human liver. J Hepatol 2001;34: 514-522.

5 Ramirez F, Pereira L. The fibrillins. Int J Biochem Cell Biol 1999;31:255-259.

6 Sakai LY, Keene DR, Glanville RW, et al. Purification and partial characterization of fibrillin, a cysteine-rich structural component of connective tissue microfibrils. J Biol Chem 1991;266:14763-14770.

7 Mir S, Wheatley HM, Hussels IE, et al. A comparative histologic study of the fibrillin microfibrllar system in the lens capsule of the normal subjects and subjects with Marfan syndrome. Invest Opthalmol Vis Sci 1998;39:84-93.

8 Visconti RP, Barth JL, Keeley FW, et al. Codistribution analysis of elastin and related fibrillar proteins in early vertebrate development. Matrix Biol 2003;22: 109-121.

9 Sakamoto H, Broekelmann T, Cheresh DA, et al. Celltype specific recognition of RGD- and non-RGDcontaining cell binding domains in fibrillin-1. J Biol Chem 1996;271:4916-4922.

10 D' Arrigo C, Burl S, Withers AP, et al. TGF- $\beta 1$ binding protein-like molecules of fibrillin-1 and -2 mediate integrin-dependent cell adhesion. Connect Tissue Res 1998;37:29-51.

11 Plaff M, Reinhardt DP, Sakai LY, et al. Cell adhesion and integrin binding to recombinant human fibrillin-1. FEBS Lett 1996;384:247-250.

12 Tiedemann K, Batge B, Muller PK, et al. Interactions of fibrillin- 1 with heparin/heparin sulfate, implications for microfibrilar assembly. J Biol Chem 2001;276: 36035-36042.

13 Ritty TM, Broekelmann TJ, Werneck CC, et al. Fibrillin-1 and -2 contain heparin-binding sites important for matrix deposition and that support cell attachment. Biochem J 2003;375:425-432.

14 Puiu L, Petrakou E, Apostolidou A, et al. Lack of Fas (APO/CD95) gene structural alterations on transcript variant ratio changes in breast cancer. Cancer Lett 2003;194:91-97.

15 Gabri C, Zurzolo C, Bifulko M, et al. Synthesis of extracellular matrix glycoproteins by a differentiated thyroid epithelial cell line. J Cell Physiol 1988;135: 39-46.

16 Miettinen M, Virtanen I. Expression of laminin in thyroid gland and thyroid tumors: an immunohistologic study. Int J Cancer 1984;34:27-30.

17 Kondo T, Nakazawa T, Murata S, et al. Stromal elastosis in papillary thyroid carcinomas. Hum Pathol 2005;36:474-479.

18 Reinhardt DP, Sasaki T, Dzamba BJ, et al. Fibrillin-1 and fibulin-2 interact and are colocalized in some tissues. J Biol Chem 1996;271:19489-19496.

19 Milewicz DM, Grossfield J, Cao SN, et al. A mutation in FBN1 disrupts profibrillin processing and results in 
isolated skeletal features of the marfan syndrome. J Clin Invest 1995;95:2373-2378.

20 Zhang $\mathrm{H}$, Apfelroth SD, $\mathrm{Hu} \mathrm{W}$, et al. Structure and expression of fibrillin-2, a novel microfibrillar component preferentially located in elastic matrices. J Cell Biol 1994;124:855-863.

21 Bhatia A, Blades S, Cambridge G, et al. Differential distribution of $\mathrm{Fc}$ gamma RIIIa in normal human tissues and co-localization with DAF and fibrillin-1: implications for immunological microenvironments. Immunology 1998;94:56-63.

22 Tseleni-Balafouta S, Gakiopoulou H, Fanourakis G, et al. Tenascin-c protein expression and mRNA splice variants in thyroid carcinoma. Exp Mol Pathol 2005, Oct 28.

23 Lacroix L, Lazar V, Michiels S, et al. Follicular thyroid tumors with the PAX8-PPARgamma1 rearrangement display characteristic genetic alterations. Am J Pathol 2005;167:223-231. 